\title{
Regulation of release factor expression using a translational negative feedback loop: A systems analysis
}

\author{
RUSSELL BETNEY, ${ }^{1}$ ERIC DE SILVA, ${ }^{2}$ CHRISTINA MERTENS, ${ }^{1}$ YVONNE KNOX, $^{1}$ J. KRISHNAN, ${ }^{2}$ \\ and IAN STANSFIELD ${ }^{1,3}$ \\ ${ }^{1}$ University of Aberdeen, School of Medical Sciences, Institute of Medical Sciences, Foresterhill, Aberdeen, AB25 2ZD, United Kingdom \\ ${ }^{2}$ Chemical Engineering and Chemical Technology, Institute for Systems and Synthetic Biology, Imperial College London, South Kensington \\ Campus, London SW7 2AZ, United Kingdom
}

\begin{abstract}
The essential eukaryote release factor eRF1, encoded by the yeast SUP45 gene, recognizes stop codons during ribosomal translation. SUP45 nonsense alleles are, however, viable due to the establishment of feedback-regulated readthrough of the premature termination codon; reductions in full-length eRF1 promote tRNA-mediated stop codon readthrough, which, in turn, drives partial production of full-length eRF1. A deterministic mathematical model of this eRF1 feedback loop was developed using a staged increase in model complexity. Model predictions matched the experimental observation that strains carrying the mutant SUQ5 tRNA (a weak UAA suppressor) in combination with any of the tested sup45 ${ }^{U A A}$ nonsense alleles exhibit threefold more stop codon readthrough than that of an SUQ5 yeast strain. The model also successfully predicted that eRF1 feedback control in an SUQ5 sup45 ${ }^{U A A}$ mutant would resist, but not completely prevent, imposed changes in eRF1 expression. In these experiments, the introduction of a plasmid-borne SUQ5 copy into a sup45 ${ }^{U A A}$ SUQ5 mutant directed additional readthrough and full-length eRF1 expression, despite feedback. Secondly, induction of additional sup45 ${ }^{U A A}$ mRNA expression in a sup45 ${ }^{U A A}$ SUQ5 strain also directed increased full-length eRF1 expression. The autogenous sup45 control mechanism therefore acts not to precisely control eRF1 expression, but rather as a damping mechanism that only partially resists changes in release factor expression level. The validated model predicts that the degree of feedback damping (i.e., control precision) is proportional to eRF1 affinity for the premature stop codon. The validated model represents an important tool to analyze this and other translational negative feedback loops.
\end{abstract}

Keywords: release factor eRF1; SUP45; negative feedback; translation; ribosome; translation termination

\section{INTRODUCTION}

Cellular protein synthesis is a highly regulated process during which ribosomes sequentially translate an mRNA template in a process that can be divided into the initiation, elongation, and termination phases. Termination is triggered when the elongating ribosome encounters an in-frame stop codon, the signal for a translation release factor to bind and release the nascent polypeptide. In eukaryotes, the release factor (RF) is a complex of two proteins: eRF1, a class I release factor that can recognize each of the three stop codons; and eRF3, an essential GTPase that stimulates the efficiency of the termination process (Frolova et al. 1994; Stansfield et al. 1995a; Zhouravleva et al. 1995). In prokaryotes, two

\footnotetext{
${ }^{3}$ Corresponding author

E-mail i.stansfield@abdn.ac.uk

Article published online ahead of print. Article and publication date are at http://www.rnajournal.org/cgi/doi/10.1261/rna.035113.112.
}

class I release factors, RF1 (recognizing UAA and UAG) and RF2 (recognizing UAA and UGA), have overlapping responsibility for recognizing the termination codons. A third, nonessential release factor, RF3, stimulates release factor removal from the ribosome following termination (Freistroffer et al. 1997; Gao et al. 2007).

The class I stop codon-recognizing release factorseRF1, RF1, and RF2 - are essential for cell viability, a reflection of the fact that efficient recognition of the stop codon is crucial for accurate protein synthesis. If the release factor fails to recognize the stop codon efficiently, caused, for example, by lowered RF cellular abundance, the extended pause will trigger at some level an alternative stop codon readthrough process (Stansfield et al. 1995b; Bertram et al. 2001). Readthrough can be caused by mis-recognition of the stop codon by a natural suppressor tRNA. In yeast, these ordinary tRNAs include the glutamine tRNA ${ }_{U U G}$ Gln and $\mathrm{tRNA}_{\mathrm{CUG}}{ }^{\mathrm{Gln}}$, which, respectively, can decode UAA and UAG via first-base G-U wobble (Pure et al. 1985; Edelman 
and Culbertson 1991). Such readthrough events will direct the synthesis of a potentially deleterious longer protein. Alternatively, longer pauses at bacterial stop codons, caused by reduced RF activity at a weak stop codon, can trigger the activity of tmRNA, a cellular emergency response to stalled ribosomes that causes the incomplete protein to be targeted for degradation (Collier et al. 2002; Hayes et al. 2002a,b; Sunohara et al. 2004).

While underabundance of release factors can allow readthrough events, their overexpression could potentially allow erroneous recognition of "sense" codons as "stop," causing synthesis of a defective truncated protein. Bacterial release factors can recognize near-cognate codons such as UGG with significant frequencies (Freistroffer et al. 2000). Furthermore, RF2 is able to trigger release of ribosomes and their associated nascent peptides in cases in which a miscognate tRNA is located in the ribosomal P-site as a result of a prior translation error (Zaher and Green 2009). Arguably, RF2 overexpression could lead to inappropriate release of nascent polypeptides.

Careful control over release factor expression is therefore needed to ensure that these potent factors are maintained at appropriate levels, preventing the accidental expression of shortened, or lengthened, polypeptides. It is thus intriguing that RF2 expression is regulated at the translational level by a negative feedback loop. The RF2 coding sequence is split into two reading frames, separated by a UGA stop codon (Craigen and Caskey 1986). The second, ORF2, is located in the +1 frame relative to ORF1. Frameshifting is necessary to translate RF2, and the efficiency of frameshifting is governed by a three-way competition between termination, frameshifting, and readthrough (Curran and Yarus 1988; Adamski et al. 1993); it is proposed that a lowered RF2 level would trigger more frameshifting, and thus, RF2 synthesis (Craigen and Caskey 1986). However, although it is assumed that this system is autoregulatory, evidence supporting this is indirect and partly derived from studies in which purified RF2 was added to in vitro translation reactions (Donly et al. 1990). The control properties of the RF2 autogenous negative feedback circuit have thus not been fully explored.

An unusual relative of this regulatory system is found in yeast, where a series of nonsense alleles of the essential yeast eRF1 gene SUP45 have been isolated, all of which are viable despite encoding a truncated eRF1 (Stansfield et al. 1996). Similar nonlethal nonsense alleles of the eRF3 gene (SUP35) have also been identified in yeast (Chabelskaya et al. 2004). The mutant SUP45 phenotypes exhibit increased nonsense suppression, which promote tRNA-mediated readthrough of the early stop codon and hence production of some reduced level of full-length release factor (Stansfield et al. 1996). eRF1 nonsense mutations of this type have been isolated in the presence of specific mutant suppressor tRNAs that help support readthrough of the premature stop codon (Stansfield et al. 1996), but intriguingly, also in wild-type tRNA backgrounds (Moskalenko et al. 2003). The level of readthrough of the premature eRF1 nonsense codons is regulated by a complex interaction between the suppressor tRNA efficiency (Stansfield et al. 1996; de Silva et al. 2010), the eRF1 protein stability and activity, and the modulatory effect of the nonsense-mediated mRNA decay system that acts to destabilize mRNAs carrying early stop codons (Chabelskaya et al. 2007; Kiktev et al. 2009). As in the case of the RF2 feedback loop, this system appears to be autoregulatory.

Expression of functional eRF1 from the nonsense alleles, as with their RF2 counterpart, depends on a translationlevel negative feedback loop. However, the eRF1 control circuit behavior relies on a straightforward two-way competition between termination and stop codon readthrough, rather than the more complex three-way competition in the bacterial RF2 circuit that additionally involves frameshifting. The simpler eRF1 case thus represents an ideal system with which to investigate the control properties of such translational negative feedback loops, the factors that influence their operation, and how robust they are to perturbation. The basic theoretical framework for a mathematical model of this system has been previously established (de Silva et al. 2010). In this study, we extend this by developing a fully parameterized mathematical model of the eRF1 translational readthrough negative feedback loop and experimentally validate the predictions of the model. We show that the model successfully represents the establishment of the feedback loop to maintain eRF1 production and thus cell viability, and quantitatively predicts the level of stop codon readthrough in these mutants. The model predicts that feedback loop constraint of eRF1 expression is regulated by the level of tRNA suppression, by the premature stop codon nucleotide context, and by the strength of the eRF1 promoter and thus level of mRNA. The experimental data, analyzed in the context of the validated model, suggest that for tight autoregulatory control, a premature stop codon in a good termination context is required.

\section{RESULTS}

\section{Parameterization of a mathematical model describing eRF1 synthesis}

A series of nonsense alleles of the yeast sup 45 gene has been isolated and characterized (Stansfield et al. 1996). The four alleles sup $45-18,-22,-28$, and -42 each carry a single premature UAA stop codon at codon positions $359,46,360$, and 411, respectively. Viability is maintained by readthrough of the premature stop codon, driven by low levels of eRF1, and by the presence of the SUQ5 gene in this genetic background. SUQ5 is a seryl tRNA gene with a mutated anticodon ( $5^{\prime}$-UUA- $\left.3^{\prime}\right)$, which creates a weak UAA suppressor tRNA. The translated release factor recognizes the 
nonsense codon on its own mRNA, thus establishing a feedback loop. In order to more effectively analyze the behavior of this translational negative feedback loop, a mathematical model had previously been developed to support an understanding of eRF1 expression (de Silva et al. 2010). This was, however, not parameterized in conjunction with experiments and had not been validated, and the effects of system perturbation had not been experimentally explored. Furthermore, the effects of different premature stop codon positions in eRF1 were not considered.

In this study, therefore, parameter values were assigned to this staged series of deterministic translation models (de Silva et al. 2010). Each model is used in turn for different stages of the parameterization process, each building in complexity over the previous one. Model 1 is used to parameterize eRF1 synthesis, Model 2 to parameterize eRF1tRNA competition at a stop codon, and Model 3 to examine the eRF1 autogenous feedback created by introducing a nonsense codon into the eRF1 reading frame itself. For simplicity, but still retaining key features, all models were coarsegrained; that is, they consolidated individual ribosome movements into a series of lumped steps, such as all elongation reactions, and the complete 5 '-leader-scanning step (Fig. 1A). A simple Model 1 of eRF1 translational synthesis was first described in which an eRF1 mRNA is translated to produce eRF1 protein (Fig. 1B). Using known values of eRF1 abundance and stability (Ghaemmaghami et al. 2003; Beyer et al. 2004), eRF1 mRNA abundance (Beyer et al. 2004), and yeast translation rate (Bonven and Gullov 1979), and assuming a steady-state condition in exponentially growing yeast, the rate of ribosomal initiation on the eRF1 mRNA was calculated so as to satisfy a steady-state eRF1 protein condition. Using the calculated ribosomal $5^{\prime}$-cap joining rate produced a ribosomal loading of seven elongating ribosomes per eRF1 mRNA, equivalent to a density of 0.53 ribosome/ $100 \mathrm{nt}$. This model prediction matches published experimental measurements of ribosome density on the eRF1 mRNA (Arava et al. 2003).

This initial model was further elaborated by incorporating a description of eRF1 mRNA synthesis and degradation. Knowing the experimentally determined half-life of eRF1 mRNA (Wang et al. 2002), the steady-state level of eRF1 mRNA (Beyer et al. 2004), and the rate of depletion of the mRNA through cell growth (a half-life of $90 \mathrm{~min}$ ), the transcriptional mRNA synthesis rate could be directly calculated (Table 3 below).

In the case of both the ribosomal initiation rate on eRF1 mRNA and that of the mRNA transcription rate, the use of this staged model development process allowed parameter values to be assigned by simple mathematical deduction. To summarize, we used a range of experimentally determined values describing eRF1 abundance and half-life, as well as SUP45 mRNA abundance and half-life, to derive the ribosomal-cap-binding rate constant for SUP45 mRNA and the rate of SUP45 mRNA synthesis.
A

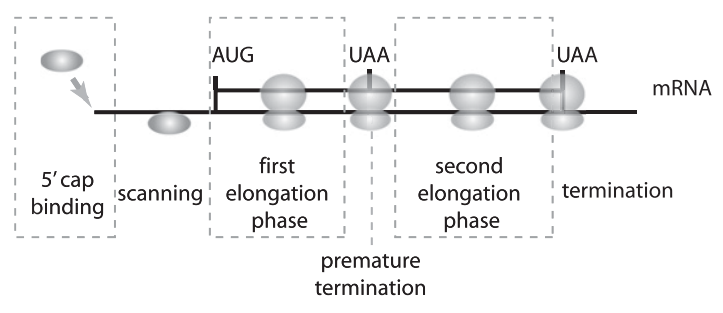

B
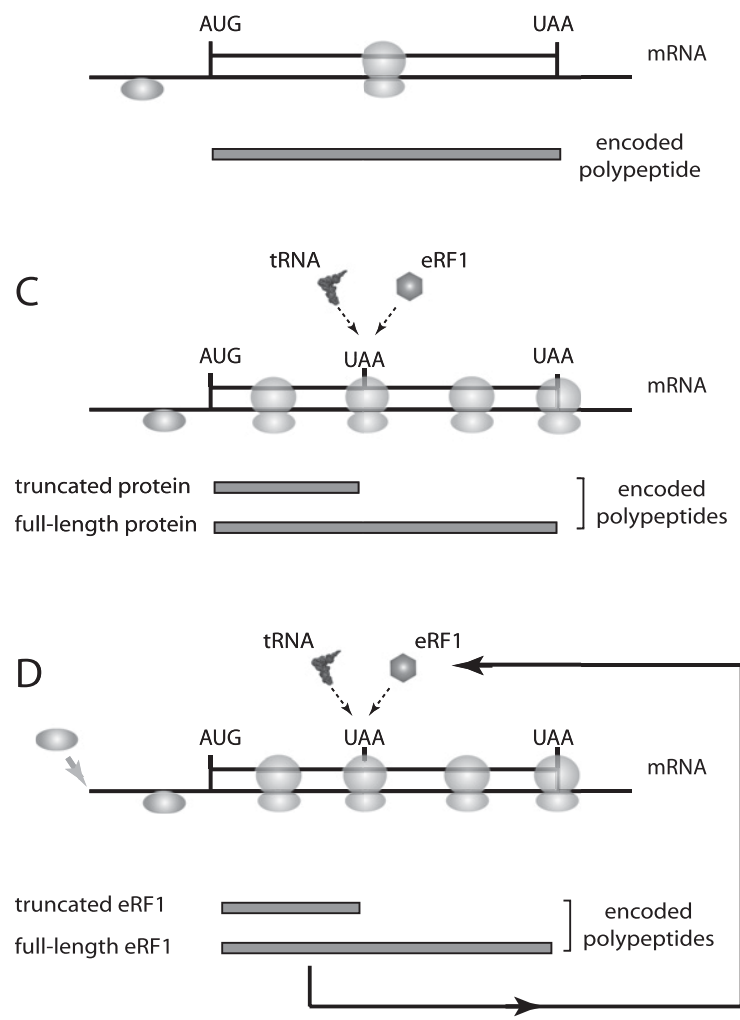

FIGURE 1. Development of a deterministic mathematical model of translation termination. Parameters were assigned to a deterministic model of translation, incorporating a description of termination, using a staged series of models of increasing complexity. (A) In each case, the complete translation process was divided into a series of modules that described a coarse-grained, or "lumped" model of the multistep translation process. $(B)$ Parameters associated with eRF1 synthesis were fitted using a simple Model 1 of eRF1 mRNA translation. $(C)$ The parameters describing release factor recognition of the stop codon and of the competition between release factor and suppressor tRNA were optimized using a more complex Model 2 in which an mRNA containing a premature stop codon is translated to produce a generic protein X. (D) A final Model 3, in which the eRF1 mRNA with a premature stop codon is translated, thus describing a negative feedback loop, was used to simulate expression of eRF1 in a cell carrying a sup45 nonsense allele.

\section{Modeling the competition between eRF1 and a suppressor tRNA}

Having established a model of the eRF1 synthesis process, Model 2 was then deployed to parameterize the competition between release factor and suppressor tRNA (Fig. 1C). 
This model describes the translation of a generic mRNA carrying a premature stop codon in the open reading frame. The SUQ5 tRNA (a weak UAA suppressor tRNA created by anticodon mutation) and the release factor compete with one another to recognize this stop codon, generating either a full-length readthrough polypeptide or a truncated protein, respectively (Fig. 1C). Since in vitro translation elongation and termination reactions display Michaelis-Menten-like kinetics (Pavlov and Ehrenberg 1996), the release factor complex, and SUQ5-tRNA elongation factor complexes were described as competing substrates for a ribosome paused at a stop codon, using standard Michaelis-Menten formulation for substrate competition for a single enzyme (Cornish-Bowden 2004) (Materials and Methods). In the absence of any previous detailed characterization of the kinetics of yeast elongation or termination, parameters from in vitro studies in Escherichia coli RF1 were used instead (Table 3 below), since the catalytic efficiency $k_{\text {cat }} / K_{\mathrm{M}}$ for RF1 is most similar to that for eRF1 in the presence of eRF3 (Eliseev et al. 2011). In addition, although RF1 and RF2 can both recognize UAA, there is some evidence that RF1 is a better terminator at UAA codons (Martin et al. 1988; Mikuni et al. 1991; Pavlov et al. 1998; Freistroffer et al. 2000) and thus may be a better model for eRF1 recognition of UAA codons. The assumption was made that the high degree of conservation of the translation process across phyla extended to the parameter values for eukaryote translation.

To provide experimental data to parameterize the model describing eRF1-SUQ5 competition, levels of readthrough (the percentage of ribosomes translating through the stop codon using suppressor tRNA activity) were experimentally measured in a cell wild type for eRF1, but carrying the SUQ5 tRNA allele, a weak UAA suppressor (BSC483/1a) (Fig. 2B). Readthrough was determined using a series of dicistronic readthrough assay vectors (Materials and Methods), which carried a UAA stop codon between the lac $Z$ and $l u c$ open reading frames. Using these vectors, a readthrough level of $3 \%$ was measured using a stop codon in a good context (the eRF1 natural position UAA stop codon) (Fig. $2 B$ ). This was consistent with earlier measurements in an SUQ5 genetic background (Stansfield et al. 1995b).

Next, SUQ5-driven readthrough of the stop codons in the sup 45 allele contexts was performed, revealing much higher levels of readthrough ranging from 5.3\% (sup45-42) to $13 \%$ (sup45-22), indicating that the sup45 allele stop codons were in less than optimal context (Fig. 2B, filled bars). This was confirmed by remeasuring the levels of readthrough of these stop codons in a wild-type, suq $5^{+}$ (nonsuppressing) genetic background, where for three of the alleles, readthrough levels were recorded that were significantly higher than the control, good context UAA codon (5.8\% for sup45-28) (Fig. 2C). This indicated that with the exception of sup45-42, the other premature stop codons were in a poor context.

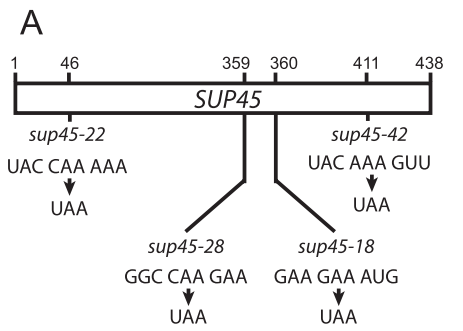

B
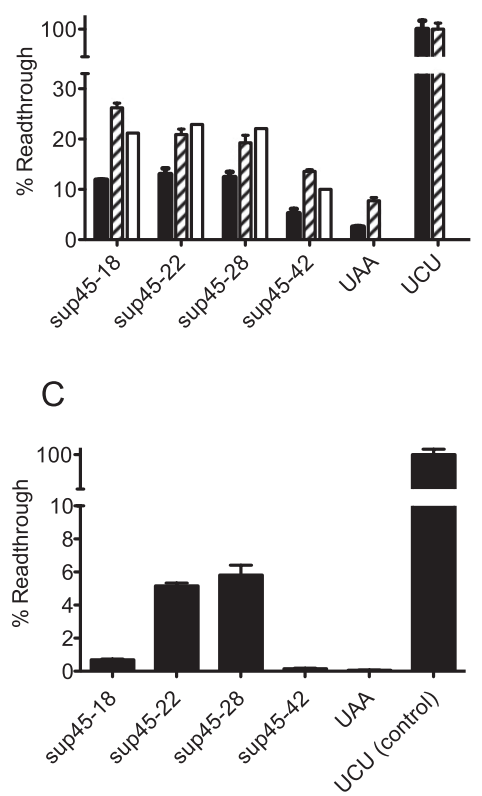

FIGURE 2. The sup45 allele premature stop codons are in nonoptimal nucleotide contexts. (A) Positions of the SUP45 allele premature stop codons and their immediate nucleotide contexts. (B) The readthrough of UAA stop codons in sup45 nonsense allele contexts was assessed using reporter assay readthrough vectors (pBET-18, -22, -28, -42, and -UAA) (Materials and Methods). Each comprised the relevant sup45 premature stop codon and its surrounding context, cloned between lac $Z$ and luc reporters. Readthrough of these stop codons was compared with that of a UAA stop codon in an optimal context, using the dicistronic plasmid reporter pBET-UAA. Readthrough assays were performed in yeast strains (BSC483/1a; SUP45 ${ }^{+}$SUQ5) carrying either a genomic SUQ5 nonsense suppressor tRNA allele (filled bars), or with a genomic SUQ5 allele and additionally transformed with a plasmid carrying an extra copy of the SUQ5 allele (pSUQ5; striped bars). Bars represent the mean of three independent transformants, \pm 1 standard deviation. To validate the mathematical model of eRF1-SUQ5 tRNA competition, Model 2 was used to simulate readthrough levels of a generic UAA stop codon in the sup45 SUQ5 [pSUQ5] transformants (open bars). (C) The UAA readthrough frequency of the premature stop codons in alleles sup45-18, $-22,28$, and -42 was assessed in a yeast strain BY4741 (wild-type suq $5^{+}$with respect to tRNA suppression), transformed with the same range of stop codon readthrough assay vectors (pBET-18, -22, -28, 42). All bars represent the mean of three independent transformants, \pm 1 standard deviation.

These in vivo measurements were then used to parameterize Model 2, in which readthrough of an early stop codon is replicated (Fig. 1C). In Model 2, the concentration of the SUQ5-tRNA-eEF1A-GTP ternary complex was adjusted 
to achieve the $3 \%$ level of readthrough experimentally measured for a stop codon in a good context, expressed in an SUQ5 yeast strain (Fig. 2). This resulted in a concentration of SUQ5 ternary complex of $0.036 \mu \mathrm{M}$, or $\sim 1000$ SUQ5 ternary complexes per cell. This value was within the expected range, albeit at the lower end, since there are $2.5 \times 10^{6}$ tRNAs per yeast cell (Waldron and Lacroute 1975), and 274 yeast tRNA genes (Percudani et al. 1997). This leads to an estimate of 9000 tRNAs expressed per singlecopy gene. However, the estimate of 1000 active SUQ5 ternary complexes is qualitatively consistent with our observation that SUQ5 is a fivefold less effective suppressor than another single-copy tRNA, SUP4 tyrosine ochre suppressor tRNA (I Stansfield, unpubl.), probably because the SUQ5 UAA suppressor tRNA is unstable or inefficiently charged.

Model 2 was then used to determine the parameters for termination at the premature stop codons in the sup45-18, $-22,-28$, and -42 alleles. Each stop codon is in a nonoptimal context that differs from that of the natural eRF1 UAA stop codon. Previous in vitro studies using bacterial termination reactions indicate that stop codon contexts affect readthrough, principally by causing an increased $K_{\mathrm{m}}$ value for the termination factor reacting with the ribosomal A-site, with $K_{\mathrm{m}}$ values varying over three orders of magnitude (Freistroffer et al. 2000). Accordingly, for each of the four sup 45 mutant allele premature stop codons, the release factor $K_{\mathrm{m}}$ value was fitted to reproduce the experimentally determined readthrough values in an SUQ5 tRNA background of $12 \%, 13 \%, 12.4 \%$, and $5.3 \%$, respectively (Fig. $2 \mathrm{~A}$ ), resulting in an allele-specific set of stop codon contextspecific affinity constants that ranged from 1.8 -fold to fivefold greater than that of release factor interaction with an optimal context stop codon (Table 3 below).

In summary, at this stage of model development, we used a Michaelis-Menten description of the termination reaction to model the competition between eRF1 and SUQ5 tRNA for the UAA stop codon. We then optimized the model value of the concentration of charged SUQ5 tRNA in the cytoplasm by matching model output to an experimentally determined value for SUQ5 readthrough in a wild-type cell. Finally, for each of the four sup 45 mutants, we adjusted the termination reaction $K_{\mathrm{M}}$ values for eRF1 reaction with the ribosome to match model output to experimentally measured values of readthrough of UAA in each of the premature codon contexts, all in an SUQ5 background.

\section{Additional expression of SUQ5 in a wild-type release factor background enhances stop codon readthrough: Model validation}

The translation release factor competes with suppressor tRNAs for stop codon binding, since both are cognate for the stop codon. Expression of a second, plasmid-borne copy of the SUQ5 gene in an eRF1 wild-type strain carrying a genomic SUQ5 allele (BSC483/1a) was expected to result in enhanced levels of nonsense suppression. The experimental data confirmed this, with the additional copy of SUQ5 raising the level of UAA readthrough by between 1.8-fold and 2.5-fold (Fig. 2B, striped bars). It is unclear why sup45-22 and -28 alleles were less SUQ5 responsive than were the sup45-18 and sup45-42 alleles. Possibly nonlinear effects may arise from the unnatural insertion of serine at the premature termination codon position, causing allelespecific altered efficiency of the full-length eRF1, or stability of the protein. It was also apparent that the sup45-18 and sup45-42 alleles were responsive to SUQ5 suppression (Fig. 2B) despite exhibiting low levels of readthrough in a wildtype, suq $5^{+}$background where UAA suppression is generated by misreading of wild-type glutamine tRNA (Fig. 2C; Edelman and Culbertson 1991). One possible explanation stems from the observation that significant codon pair bias exists in several genomes, including the yeast genome (Buchan et al. 2006). Certain codon pairs are thus favored, possibly due to negative and positive stearic interactions between different types of tRNA when juxtaposed in the ribosomal P- and A-sites (Buchan et al. 2006). It is notable that both sup45-18 and sup45-42 premature stop codons are preceded by UAC tyrosine codons (Fig. 2A). It is thus possible that misreading of a UAA stop codon by a tRNA $_{\text {UUG }}^{\text {Gln }}$ is not favored when there is a tyrosyl tRNA in the P-site, leading to poor levels of readthrough in a wildtype tRNA background. However, the seryl tRNA SUQ5 may interact more effectively with A-site UAA despite a tyrosyl P-site tRNA, leading to effective SUQ5 suppression.

The establishment of this data set allowed Model 2 prediction of eRF1-SUQ5 competition to be validated. The level of SUQ5 defined in Model 2 was accordingly doubled to mimic the expression of the extra tRNA gene copy. The model was then used in turn to predict stop codon readthrough at each of the four different sup 45 premature stop codon types, each with a different context and thus termination efficiency. This was achieved for each mutant by varying the termination $K_{\mathrm{m}}$ constant in each case (Table 3 below). The comparison of the experimental data with that of the Model 2 prediction shows clearly that the model closely matches the experimentally determined approximately twofold increase in readthrough observed in a wildtype eRF1 background upon the introduction of a second, plasmid-borne copy of the SUQ5 gene (Fig. 2B, open bars). Model 2 was thus able to closely mimic experimentally measured eRF1-SUQ5 competition occurring at premature stop codons in nucleotide contexts dictating a range of termination efficiencies.

\section{A model of a translational negative feedback loop correctly predicts the sup45 allosuppressor phenotype}

Having established a model of stop codon recognition in the context of mRNA translation and successfully validated 
the eRF1-tRNA competition, it was then possible to reassign the identity of the mRNA-encoded protein in Model 3 to eRF1, effectively establishing a negative feedback loop in which eRF1 recognizes the premature stop codon on its own mRNA (Fig. 1D). Whereas Model 2 describes a generic mRNA containing a premature stop codon (directing synthesis of an unspecified protein), Model 3 mRNA carries a premature stop codon and encodes eRF1. It thus describes the eRF1 nonsense alleles, in which the eRF1 translation product feeds back to inhibit eRF1 production through premature stop codon recognition, in competition with the readthrough-promoting SUQ5 tRNA.

This final iteration of the model also incorporated a description of the effect of nonsense-mediated decay (NMD) on the level of eRF1 mRNA stability, through which the presence of a premature stop codon within an mRNA triggers its enhanced degradation (Losson and Lacroute 1979). The extent of this destabilization is dictated by the nonsense codon position, with more $5^{\prime}$ positions being more destabilizing (Losson and Lacroute 1979). The NMD pathway is known to affect the behavior of some eRF1 premature stop codon mutants (Chabelskaya et al. 2007), and it was therefore important to take this effect into account when developing a model to describe the behavior of these mutants. The position effect of premature nonsense codons has been experimentally determined with some accuracy (Cao and Parker 2001), and these data were used to define an NMD destabilization function (Materials and Methods, Eqs. 3 and 4). Stop codon readthrough antagonizes NMD (Losson and Lacroute 1979; Keeling et al. 2004), and this effect was incorporated into the model so that mRNA stability was dynamically modulated in response to variations in nonsense suppression level.

To validate this model of the feedback loop, it was first necessary to measure UAA readthrough in all four sup45 nonsense alleles. Accordingly, in each of the four sup45 mutants (all carrying the genomic SUQ5 suppressor allele), readthrough levels were experimentally determined using the stop codon readthrough vector carrying a UAA stop codon in that mutant's specific context. The results showed that, as expected, the introduction of a sup45 nonsense allele into an SUQ5 genetic background produces a clear allosuppressor effect, i.e., nonsense suppression is considerably enhanced (Stansfield et al. 1995b, 1996). Readthrough levels as high as 38\% (sup4522) were recorded (Fig. 3A, filled bars), an almost threefold increase over levels of readthrough of this stop codon in a SUP45 $5^{+}$SUQ5 background (Fig. 2).

Using this data set, it was then possible to validate Model 3 , representing the eRF1 negative feedback loop. For each of the four mutants, Model 3 was programmed using the termination $K_{\mathrm{M}}$ affinity constant appropriate for each of the mutants (Table 3 below) and the level of stop codon readthrough predicted. The results (Fig. 3B, filled bars) show a close convergence between the model prediction and the experimental data, thus validating the ability of Model 3 to predict the enhanced suppressor phenotype generated by the establishment of a negative feedback loop in each of the mutants. To further analyze the model, we
A

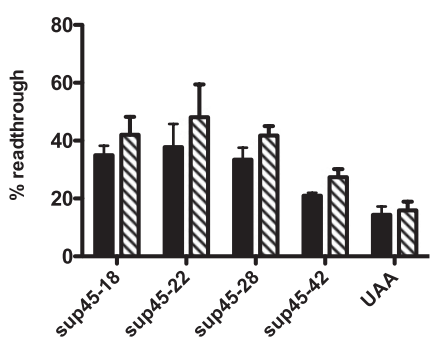

B

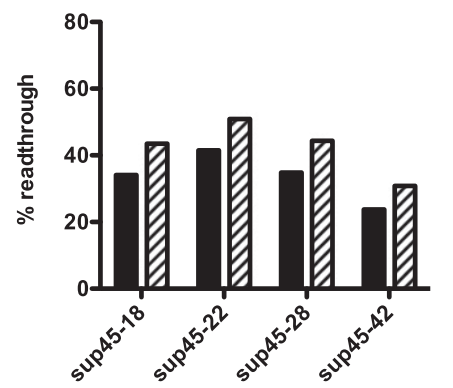

C

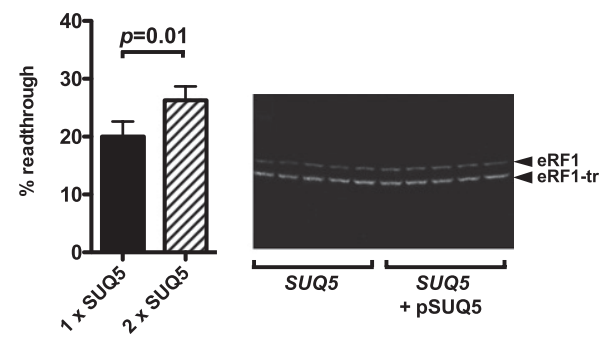

D

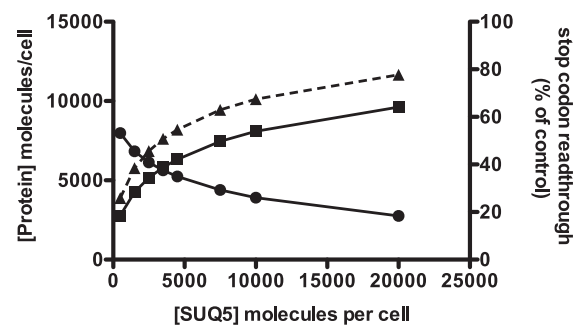

FIGURE 3. Stop codon readthrough in the sup 45 nonsense alleles responds to perturbation by increased levels of suppressor tRNA. (A) Using the galactosidase-luciferase dicistronic reporter assay, stop codon readthrough (UAA codon) was assessed in four eRF1 nonsense allele yeast strains with a sup45 SUQ5 genotype (filled bars), as well as in sup45 SUQ5 [pSUQ5] transformants (striped bars), in which the level of SUQ5 tRNA had been supplemented by transformation with a plasmid-borne copy of the SUQ5 gene encoding an ochre suppressor seryl-tRNA. Bars represent the mean of three independent transformants \pm 1 standard deviation. (B) A mathematical model of the eRF1 translational negative feedback loop was used to simulate readthrough levels in the sup45 SUQ5 mutant backgrounds (filled bars), as well as in the sup45 SUQ5 [pSUQ5] transformants (striped bars), in which SUQ5 tRNA gene copy number was doubled. $(C)$ Using Western blot analysis with polyclonal anti-eRF1 antibody, the levels of eRF1 premature stop codon readthrough were directly quantified in the sup45-42 SUQ5 mutant with, and without, an extra plasmid-borne SUQ5 gene copy. Expression of full-length and truncated eRF1 was quantified in five independent transformants of each type using a CCD camera, and readthrough levels calculated and significance tested using a $t$-test. $(D)$ Model prediction of the response of the sup 45 feedback loop to increases in SUQ5 suppressor tRNA level, showing predicted levels of eRF1 (ם), truncated eRF1 ( ), and readthrough $(\boldsymbol{\Lambda}$, dashed line). 
carried out sensitivity analysis for all parameters with reference to the experimentally measured value of stop codon readthrough in the sup45-18 SUQ5 genetic background, confirming that sensitivity was at its minimum at the optimized parameter values (Supplemental Fig. S1). We further carried out responsiveness analysis for all parameters (Supplemental Fig. S2), showing that, as expected, the model was most responsive to parameter values that control ribosomal A-site competition between suppressor tRNA and eRF1, to parameter values that regulate the level of sup45 mRNA, and its translatability by ribosomes (the rate of ribosomal interaction with the $5^{\prime}$ cap).

\section{Perturbation of the sup45 negative feedback loop by additional SUQ5 suppression}

To provide further evidence of the predictive power of the model, the experimental system was perturbed by expressing an additional copy of the SUQ5 gene in each of the four different sup45 SUQ5 genetic backgrounds. As before, levels of stop codon readthrough were recorded for stop codons in the appropriate context, revealing that the enhancement of readthrough by the extra tRNA suppressor copy only increased between 1.1-fold and 1.3-fold (Fig. 3A, hatched bars), rather than the approximately twofold to 2.3-fold increase seen in a wild-type SUP45 strain. Clearly the feedback loop limits the ability of SUQ5 tRNA to drive readthrough. This effect was confirmed by measuring the ratio of full-length eRF1 to total expressed eRF1 (truncated plus full-length) in the sup45-42 mutant using Western blot analysis and an eRF1 antibody (Fig. 3C). Western-blot eRF1 readthrough measurements were made in a sup45-42 SUQ5 strain and compared with the same estimate of percent readthrough in a sup45-42 SUQ5 [pSUQ5] strain expressing an extra ectopic copy of the suppressor tRNA. Using five independent transformants, the results show that an additional copy of SUQ5 only drives a modest increase in readthrough from $20 \%$ to $26.3 \%$. This significant $(p=$ 0.01 ) increase is nevertheless much less than the approximately twofold increase in readthrough observed when an extra copy of SUQ5 tRNA is introduced into a wild-type $S U P 45^{+}$background, which lacks the release factor feedback loop (Fig. 2B, bars labeled "UAA"). By measuring eRF1 mRNA readthrough directly using an antibody against eRF1 protein, we could thus demonstrate that the increase in sup45-42 premature UAA codon readthrough (from $20 \%$ to $26.3 \%$ ) driven by the extra copy of SUQ5 was very similar to the increase in readthrough levels measured using the dicistronic reporter assay in the same genetic backgrounds (increased from $21 \%$ to $27.3 \%$ ) (Fig. 3A). The dicistronic readthrough assay using reporter genes is thus an excellent proxy for the actual eRF1 UAA stop codon readthrough event.

Using this experimental perturbation, it was again possible to validate the predictions of Model 3. For each of the four mutants, the termination reaction affinity constant appropriate for each of the mutants was incorporated into Model 3 (Table 3 below), then the concentration of SUQ5 was doubled in the model to mimic the expression of an extra copy of SUQ5. The level of stop codon readthrough predicted by the model was then recorded. The results (Fig. $3 \mathrm{~B}$, hatched bars) again show a close convergence between the model prediction and the experimental data (Fig. $3 \mathrm{~A}, \mathrm{C})$, thus validating the ability of Model 3 to predict the response of this translational negative feedback loop to perturbation by suppressor tRNA.

The validated Model 3 was therefore used to examine more comprehensively the effect on the feedback loop of applying increasing amounts of SUQ5 tRNA to the system. The results (Fig. 3D) showed that gradually increasing levels of the tRNA to drive readthrough caused consequential increases in the level of full-length eRF1, and gradual increases in stop codon readthrough as seen experimentally (Fig. 3A,C). The model clearly predicts that in the sup45 nonsense allele backgrounds, increasing the concentrations of suppressor tRNA does not increase readthrough to the same extent as it does in a wild-type SUP $45^{+}$background.

\section{Overexpression of mutant sup $45^{U A A}$ mRNA results in additional production of eRF1 despite negative feedback loop action}

Negative feedback control mechanisms can exert varying degrees of control over a system, depending on the structure of the feedback mechanism and its control properties. In some cases, feedback exerts a strict control regime to maintain a precise system set point, while in other cases, negative feedback serves only to "damp" or partly resist system perturbations.

One common type of perturbation to which any gene expression regulatory mechanism is exposed is altered mRNA expression, generated by stochastic bursts of transcriptional activity, which inject extra copies of an mRNA into the system. It was therefore of interest to examine how the sup $45^{U A A}$ negative feedback loop would respond if levels of its mRNA expression were artificially increased under the control of a regulatable promoter. Would the concentration of eRF1 be maintained at a precise set-point, or would levels of eRF1 increase? Would any "damped" response be apparent, i.e., an increase, but limited by the feedback loop? Thus, the response of the feedback loop to this type of perturbation could reveal to what extent the feedback loop was autoregulatory, i.e., whether the system set point was changed by the injection of additional sup45 nonsense allele mRNA. Accordingly, a doxycycline-regulated expression system (Gari et al. 1997) was used to control expression of an ectopic copy of either the wild-type SUP45 gene or the sup45-18 nonsense allele. Coincident with this, steady-state levels of stop codon readthrough were recorded, and Western blots were used to analyze full-length eRF1 expression. 
Initially, either the SUP45 wild-type allele or the sup4518 allele was expressed in a wild-type SUP45 background in either the absence (eRF1 expression on) or presence of doxycycline (eRF1 expression off). As expected, readthrough levels were unchanged (Fig. 4A), a reflection of the fact that (1) the genomic copy of SUP45 was wild type, and (2) overexpression of eRF1 alone is not sufficient to generate antisuppression (coincident eRF3 overexpression is also required) (Stansfield et al. 1995a).

In successive experiments, either the wild-type allele or the sup45-18 allele was again expressed under doxycycline
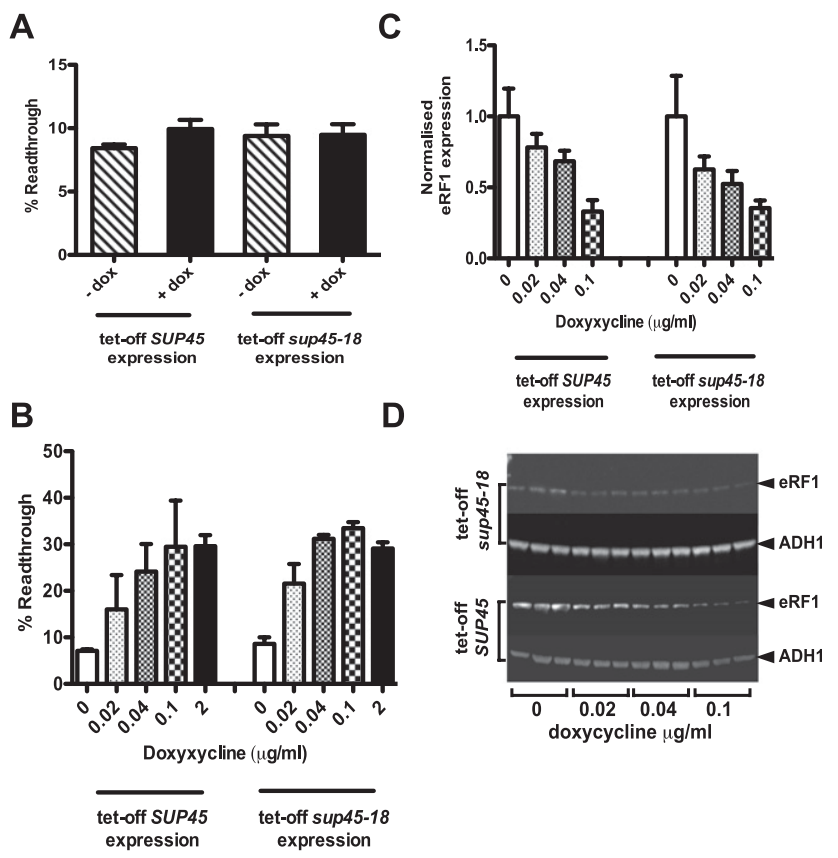

FIGURE 4. Stop codon readthrough in the sup45 nonsense alleles responds to perturbation by increased levels of SUP45 mRNA engineered using a doxycycline regulatable promoter. $(A)$ Using the dicistronic reporter gene assay carrying a UAA stop codon in the sup45-18 context (pBET-18), UAA stop codon readthrough was assessed in a yeast strain wild-type for eRF1, but carrying the SUQ5 suppressor tRNA, and transformed with either plasmid pMER or pMER-18. These express, respectively, the SUP45 or sup45-18 alleles under control of a tet-off promoter that allows the system to be perturbed by inducing the expression of extra copies of the respective mRNAs, and responses measured. Transformants were grown in the absence (striped bars) or presence (filled bars) of $2 \mu \mathrm{g} / \mathrm{mL}$ doxycyline. Bars represent the mean of three independent transformants \pm 1 standard deviation. (B) pMER (allowing doxycycline-regulated expression of the SUP45 allele) or pMER-18 (allowing doxycyclineregulated expression of the sup45-18 allele) was transformed into a sup45-18, SUQ5 yeast strain. Readthrough of a UAA stop codon in the sup45-18 premature stop codon context was assessed using readthrough assay vector pBET-18. Cultures were grown in a range of doxycycline concentrations to regulate Sup45p expression, and readthrough levels were quantified. Levels of full-length eRF1 were also quantified $(C)$ in the same mutant using the tet-off regulated expression of eRF1 described above, using quantitation of Western blot analysis $(D)$. Blots were probed with both anti-eRF1 antibody and protein loadings normalized using anti-alcohol dehydrogenase 1 (Adhlp) antibody as a constitutively expressed control protein. control, this time in a sup45-18 SUQ5 genetic background. The results clearly show that the wild-type SUP45 allele, when expressed under doxycycline control, is able to complement the sup45-18 mutant phenotype, with the levels of stop codon readthrough diminishing as doxycycline is progressively removed and eRF1 expression is switched on (Fig. 4B). However, unexpectedly, ectopic expression of the mutant sup45-18 allele also complemented the host mutant phenotype; doxycycline removal, and thus full sup45-18 expression, caused the same reduction in stop codon readthrough as did the wild-type allele (Fig. 4B). Clearly the extra copies of the sup45-18 allele were able to drive expression of more full-length eRF1, even in the context of the negative feedback loop. This was confirmed by measuring the levels of full-length eRF1 using Western blot analysis (Fig. 4D). The quantification of these Western blots showed clearly that as expression of the sup45-18 mRNA is increased, the level of expression of full-length eRF1 also increases (Fig. 4C). The relative levels of both Sup45-18p and truncated Sup45-18p driven by the doxycycline promoter could not be quantitatively determined in this study, since eRF1 cannot be $\mathrm{N}$-terminally tagged, C-terminal tagging can influence termination efficiency (I Stansfield and R Betney, unpubl.), and a monoclonal antibody against the $\mathrm{N}$-terminal portion of eRF1 was not available. Nevertheless, the use of a polyclonal antibody recognizing the full-length eRF1 protein showed that the qualitative model predictions were validated by experiment, with raised levels of the sup 45 nonsense allele mRNA driving production of more full-length eRF1 despite feedback effects (Fig. 4B-D).

We then examined whether the mathematical model was able to predict the ability of overexpressed sup45-18 allele to complement its own phenotype. Using Model 3, the expression level of mutant eRF1 mRNA was increased in a stepwise fashion, and levels of eRF1 full-length and truncated protein were recorded. The simplifying assumption was made that inducing the mRNA using the tet-off promoter system did not alter mRNA half-life, since half-life is normally an intrinsic property of the mRNA, rather than of its expression level. The modeling shows clearly that as expression levels of the mRNA increase, so the levels of full-length eRF1 increase, and the level of readthrough gradually falls (Fig. 5).

Since many of the sup 45 alleles analyzed carried premature stop codons in a poor context, it seemed probable that this would influence feedback responses to perturbation. Accordingly, the validated model was used to investigate the effect of changing the affinity of full-length eRF1 for the premature stop codon in the context of the negative feedback circuit. The model clearly predicted that a tighter control of the autogenous feedback loop would be achieved if the premature stop codon were in a good context, described by a smaller $K_{\mathrm{M}}$ affinity constant for eRF1 recognition of the premature termination codon (Fig. 6); when the $K_{\mathrm{M}}$ is lowered, the system is more resistant to increases in expression of the mutant allele mRNA, evidenced by the 

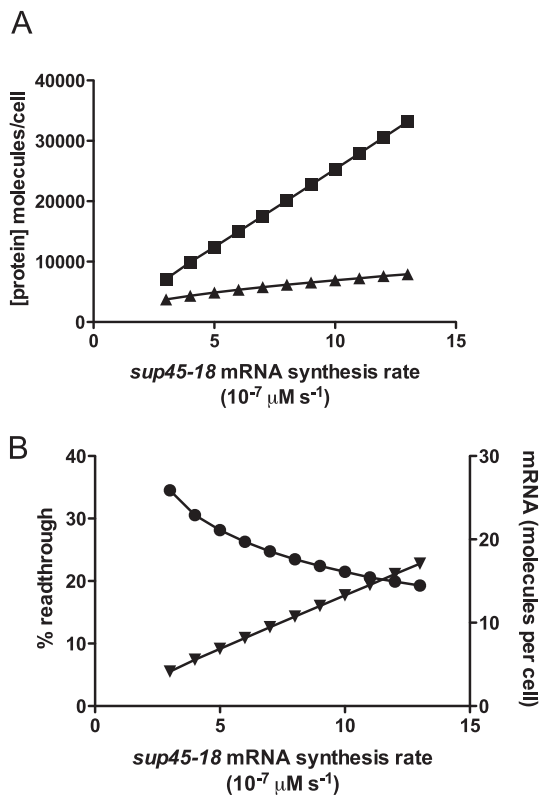

FIGURE 5. Modeling the consequences of artificially overexpressing a $\sup 45^{U A A}$ allele at the transcriptional level; feedback restricts, but does not eliminate, additional eRF1 expression. (A) The increased transcription rate of sup45-18 mRNA that was experimentally engineered using the tet-off regulatable promoter system (Fig. 4) was replicated using Model 3, revealing that increased transcription of the sup $45^{\text {UAA }}$ allele causes enhanced production of (⿴) truncated eRF1 (eRF1-tr), but proportionately less eRF1 production $(\mathbf{\Lambda})$ as the mRNA synthesis rate is raised. (B) Increased production of sup4518 mRNA $(\boldsymbol{\nabla})$ results from increased transcription rates, and levels of stop codon readthrough $(\mathbf{)})$ decrease as sup45-18 mRNA translation produces more truncated eRF1 (eRF1-tr), but disproportionately less full-length eRF1.

less steep gradient of the response. Thus when the affinity constant is lower, the feedback loop resists increases in sup 45 nonsense allele expression level, although testing this experimentally was beyond the scope of the present study.

\section{DISCUSSION}

Negative feedback loops play crucial roles in homeostasis throughout biology. In this study, the properties of one such feedback example were examined because it represents an unusual example of negative feedback operating at the level of translation. Nonsense mutations in the essential SUP45 gene encoding the release factor eRF1 are not, as would be expected, lethal, but instead form a viable eRF1 expression system in which levels of eRF1 are maintained by significant levels of stop codon readthrough (Stansfield et al. 1996; Moskalenko et al. 2003). However, the properties of the eRF1 feedback loop and its response to perturbation have not previously been investigated.

There are significant similarities between this mutant system, and the natural bacterial release factor RF2 autogenous control system. RF2 is encoded by an mRNA carrying a reading frame in two parts, the second arranged in the +1 frame relative to the 0 frame of the first. A UGA stop codon, recognizable by RF2, is found at the +1 frameshift point. If sufficient RF2 is present in the cell, ribosomes arriving at this premature UGA codon are recognized by RF2 to trigger termination and the synthesis of a truncated, nonfunctional peptide. If the abundance of RF2 drops, cis elements within the RF2 mRNA encourage a +1 frameshift event, resulting in translation of the second reading frame, and the synthesis of more RF2, hence generating an autoregulatory system. Thus both RF2, and the mutant sup45 system described in this study depend on feedback recognition of a stop codon by a release factor on their own mRNA. Release factor production is in both cases governed by the competition between fulllength release factor, and a stop codon bypass functioneither suppressor tRNA or frameshifting, respectively, for eRF1 or RF2 nonsense alleles. However, in the case of the RF2 premature UGA codon, a third alternative event, namely, stop codon readthrough, generates a three-way competition between readthrough, termination, and frameshifting. In the case of eRF1, the simpler nature of two-way competition between termination and SUQ5 tRNA-driven readthrough recommends it as a better tool to probe the control properties of a translation autoregulatory loop. Earlier attempts to deduce RF2 loop behavior also used simple models of the control circuit, validated by experiments using UAG suppressor tRNAs to drive readthrough of a UAG codon (recognized by RF1, but not RF2) located at the center of an otherwise wild-type RF2 frameshift "window" in a reporter gene construct. These showed unequivocally the three-way competition at the frameshift site (Curran and Yarus 1988). However, this same study did not address how UGA suppressor tRNAs might perturb the endogenous RF2 expression (Curran and Yarus 1988).

In this study, we sought to address these gaps in knowledge by measuring stop codon readthrough using ectopic reporters, and also endogenous eRF1 protein expression in sup 45 nonsense alleles with, and without, system perturbation through

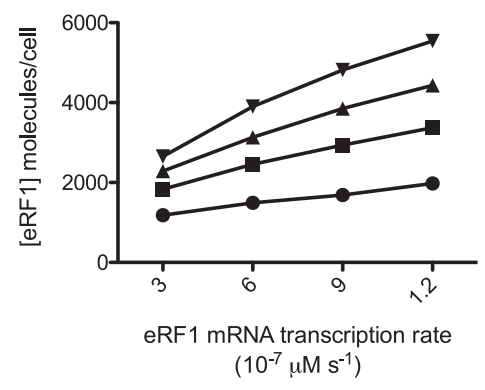

FIGURE 6. Premature stop codon contexts well-recognized by eRF1 allow efficient feedback control of eRF1 and resist perturbation through enhanced transcription. Model 3, describing the regulation of eRF1 expression in the sup45 SUQ5 mutants, was used to simulate the effect of increased transcription of the nonsense sup 45 allele on eRF1 expression levels, with varying affinity of the release factor for the premature stop codon. $\left(K_{\mathrm{M}}=0.002 \mu \mathrm{M} ;(\mathbf{\square}) K_{\mathrm{M}}=0.008 \mu \mathrm{M}\right.$;

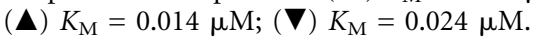


tRNA expression, and overexpression of the eRF1 encoding mRNA. Adding extra copies of the SUQ5 tRNA to a wildtype $S U P 45^{+}$strain already carrying a genomic SUQ5 allele caused the expected approximately twofold increase in stop codon readthrough, since suppressor tRNA gene copy level had doubled. This competition between the release factor apparatus and a suppressor tRNA was accurately predicted by Model 2 (Fig. 2B). The further development of Model 3, used to represent the negative feedback loop found in the sup45 nonsense alleles, correctly predicted the allosuppressor phenotype that typifies this class of mutation (Fig. 3), i.e., the enhancement of weak tRNA suppressor activity by the sup 45 nonsense alleles. Furthermore, the model was able to accurately report the experimentally observed effect of adding an extra copy of the SUQ5 tRNA into the nonsense allele genetic backgrounds. This perturbation of the system by extra suppressor tRNA, perhaps surprisingly given the autoregulatory nature of many negative feedback loops, caused some increase in readthrough (1.3-fold) and matching increases in levels of full-length eRF1 (Fig. 3A,C). This was less than the 2.3 -fold readthrough increase seen in a $S U P 45^{+} S U Q 5$ strain transformed with an extra SUQ5 copy (i.e., no feedback circuit) measured at a stop codon with the sup45-42 context (Fig. 2A). This experiment clearly showed that although the feedback loop did not maintain eRF1 expression at a precise set point, it was nevertheless exerting a subtle damping effect on the system in response to raised concentrations of suppressor tRNA. There was thus a "resistance to change" exerted by the sup 45 feedback.

The damping action of the eRF1 negative feedback loop was also seen when expression of the sup45-18 allele was increased using a regulatable promoter to drive expression of an ectopic copy of this mutant allele. This perturbation caused a marked decrease in stop codon readthrough, both when the wild-type allele was expressed, but also when the sup $45^{\text {UAA }}$ allele was expressed, the latter a more unexpected result (Fig. 4). However, this $\sup 45^{U A A}$ allele behavior was also replicated by the model (Fig. 5). The explanation of why this should be so is as follows. As the level of sup $45^{\text {UAA }}$ mRNA increases, stop codon readthrough drives the production of more full-length eRF1. However, the level of SUQ5 suppressor tRNA, the competitor species, is fixed. Thus with SUQ5 concentrations fixed, but greater amounts of sup $45^{U A A}$ mRNA driving increased eRF1 synthesis, levels of readthrough slowly decrease as mRNA concentrations are gradually increased. In other words, as termination becomes increasingly dominant over readthrough, disproportionately more eRF1-tr is produced, and disproportionately less full-length eRF1, thus readthrough levels decrease. The system response is therefore damped by feedback.

The mathematical model produced as part of this study is able to successfully predict quantitatively the response of the experimental system to perturbation in several ways. For instance, the model correctly predicts that combining a sup $45^{U A A}$ allele with SUQ5 produces the allosuppressor phenotype observed in vivo, i.e., that a combination of a sup $45^{\text {UAA }}$ allele and SUQ5 causes an enhancement of SUQ5 suppressor activity. The model correctly predicts that doubling the concentration of the SUQ5 tRNA in a sup $45^{\text {UAA }}$ SUQ5 strain only moderately increases readthrough, rather than doubling it as occurs in a SUP45 ${ }^{+}$strain (evidence of feedback damping). Finally, the model correctly predicts that overexpressing the mutant $\sup 45^{U A A}$ allele using a regulatable promoter will cause some reductions in readthrough, but also increases the expression level of full-length eRF1. The model behavior thus closely reproduces biological function, i.e., the responses of different perturbations to the information flow in the feedback circuit are in agreement with the model. We are therefore confident that the nature and control properties of the eRF1 feedback circuit is well represented by the model structure.

This damping response was qualitatively predicted by the model (Fig. 5). Considering that the copy number of SUQ5 remained constant in these experiments, it implies that readthrough levels decreased due to increases in eRF1 expression relative to the (fixed) SUQ5 level, and in fact, this was confirmed by Western blot analysis (Fig. 4C,D). Indeed, the model also predicts that increased mRNA sup4518 levels will enhance full-length eRF1 expression, which consequently decreases readthrough (Fig. 5). The increase in eRF1 levels caused by translation of the additional mRNA copies is substantially limiting its own production by favoring early termination at the premature stop codon, i.e., reduced readthrough.

Thus, evidence of a feedback "damping" effect can be seen in the case in which extra suppressor tRNA is injected into the system, causing a less than proportional increase in readthrough (Fig. 3) and, separately, under conditions in which SUQ5 levels remain constant, but eRF1 mRNA concentration is increased (Fig. 4). These latter conditions are precisely those in which a feedback loop might be expected to act as a feedback regulator of eRF1 expression in the cell, for example, to control transcriptional bursts, which are known to occur in some gene expression systems (Golding et al. 2005). Moreover, application of the final model predicts clearly that enhanced levels of control of the system in response to perturbation (for instance, by transcriptional up-regulation of the nonsense allele sup45) can be achieved if there is a greater affinity of release factor for its premature stop codon, for instance, if the stop codon is in a better context (Fig. 6). This finding has implications for the control properties of the RF2 circuit, where evidence suggests that the premature UGA stop codon is, in fact, in a poor context (UGAC) (Poole et al. 1995; Major et al. 1996).

Overall, this study has documented the development and validation of a detailed model of an unusual example of autogenous control of a translation factor in a eukaryote. The parameterization of this model and its use to predict the effects of experimental perturbation allowed its successful validation using an extensive series of in vivo experi- 
mental data sets. Analysis of the model and validation of its predictions revealed that in this translationally regulated system, negative feedback does not act to tightly regulate eRF1 expression at a particular set-point range of values, but rather acts as a more subtle damping mechanism that may serve to restrict noise in gene expression. The model represents a flexible platform for the further exploration of this system and for the use of translational feedback control in synthetic biology, as well as investigations of other translationally regulated systems such as the bacterial RF2 and IF3 autogenous control circuits (Betney et al. 2010).

\section{MATERIALS AND METHODS}

\section{Strains and media}

Yeast strains were grown at $30^{\circ} \mathrm{C}$ in either YPD complete medium $(1 \%[\mathrm{w} / \mathrm{v}]$ yeast extract, $2 \%[\mathrm{w} / \mathrm{v}]$ bacto-peptone, $2 \%[\mathrm{w} / \mathrm{v}]$ glucose) or synthetic defined (SD) minimal medium $(0.67 \%$ [w/v] yeast nitrogen base without amino acids, $2 \%$ [w/v] glucose) supplemented with the appropriate amino acids and nucleotides. Release factor mutant strains (sup45-18, -22, -28, and -42), and the progenitor wild-type BSC483/1a (Table 2 below) were described previously (Stansfield et al. 1996). To facilitate genetic manipulation of these strains, the LEU2 gene was deleted using PCRmediated targeted gene knockout using the NatMX4 selectable marker, using primers BSC483/1a LEU2::NatMX-f and BSC483/ 1a LEU2::NatMX-r (Table 1), creating BSC483/1a Dleu2, and corresponding $\Delta l e u 2$ variants for each of the four different sup45 mutants (Table 2).

E. coli strain XL1-Blue (recA1 endA1 gyrA96 thi-1 hsdR17 supE44 relA1 lac[F' proAB lacIqZAM15 Tn10(Tetr)]) was used for gene cloning and plasmid propagation using standard methods as described (Sambrook and Russell 2001).

\section{Plasmid construction}

Readthrough assay plasmids to measure readthrough of each of the sup45 premature stop codons, each in its own nucleotide context, were created in the pAC98 plasmid (derived from pAC74) (Stahl et al. 1995) using several primers shown in Table 1 and using methods as described (Williams et al. 2004). Plasmid pAC98 comprises a constitutively expressed translational fusion of a lac $Z$ gene with a firefly luciferase $l u c$ gene, separated by a unique NotI restriction site. Pairs of primers SUP45-18-F and SUP45-18-R, SUP45-22-F and SUP45-22-R, SUP45-28-F and SUP45-28-R, SUP45-42-F and SUP45-42-R, and UAA-F and UAA-R were annealed and ligated into the unique NotI site to create pBET$18,-22,-28,-42$, and -UAA, respectively. They encoded, respectively, the premature termination codons in their natural contexts from the sup45-18, -22, -28, and-42 alleles (with 12 nt of sequence both $5^{\prime}$ and $3^{\prime}$ of the premature stop codon), and the natural stop codon from the yeast SUP45 gene. Primers SUP45-UCU-F and SUP45-UCU-R were annealed and ligated into pAC98 to created pBET-UCU, in which a UCU serine codon was cloned in between the $l a c Z$ and $l u c$ genes, representing a $100 \%$ readthrough control vector.

Plasmids pMER and pMER-18 comprised the wild-type SUP45 gene and the sup45-18 allele, respectively, cloned into the pCM189 plasmid, which allows doxycycline-regulated control of gene expression (Gari et al. 1997). The SUP45 or sup45-18 genes were PCR-amplified from BSC483/1a $\Delta$ leu2 or sup45-18 $\Delta$ leu2, respectively, using primers pMER-F and pMER-R (Table 2). The amplified fragment was then cloned into PCM189, and confirmed by sequencing.

A plasmid (pSUQ5) carrying the SUQ5 UAA-suppressor tRNA (Waldron et al. 1981) was created by amplifying the SUQ5 tRNA gene from genomic DNA of strain BSC483/1a $\Delta$ leu2 using primers SUQ5-tet15 and SUQ5 tetrev, and ligating into vector pRS415 with the help of SpeI and XhoI restriction enzymes (Sikorski and Hieter 1989).

TABLE 1. Primers used in this study

\begin{tabular}{|c|c|}
\hline Primer & Sequence $5^{\prime}-3^{\prime}$ \\
\hline sup45-18 F & GGCCTACAGGGCCCGCTACCGGCCAATAAATGGACGTTGTCGAATGGA \\
\hline sup45-18 R & ATGTCCCGGGCGATGGCCGGTTATTTACCTGCAACAGCTTACCTCCGG \\
\hline sup45-22 F & GGCCTACAGGGCCСATTCCACTGTACTAAAAAATGTTAACAGAATGGA \\
\hline sup45-22 R & ATGTCCCGGGTAAGGTGACATGATTTTTTACAATTGTCTTACCTCCGG \\
\hline sup45-28 F & GGCCTACAGGGCCCAAAGCTACCGGCTAAGAAATGGACGTTGAATGGA \\
\hline sup45-28 R & ATGTCCCGGGTTTCGATGGCCGATTCTTTACCTGCAACTTACCTCCGG \\
\hline sup45-42 F & GGCCTACAGGGCCСATGCTGCGTTACTAAGTTAATTTTGAAGAATGGA \\
\hline sup45-42 R & ATGTCCCGGGTACGACGCAATGATTCAATTAAAACTTCTTACCTCCGG \\
\hline sup45 UAA F & GGCCTACAGGGCCCTATGATTTCATTTAAATAAATAAAAGGGAATGGA \\
\hline sup45 UAA R & ATGTCCCGGGATACTAAAGTAAATTTATTTATTTTCCCTTACCTCCGG \\
\hline sup45 UCU F & GGCCTACAGGGCCСGСТACCGGCCAATCTATGGACGTTGTCGAATGGA \\
\hline sup45 UCU R & ATGTCCCGGGCGATGGCCGGTTAGATACCTGCAACAGCTTACCTCCGG \\
\hline pMER F & TCAATTCGGGGGATCCCAATAATGGATAACGAGGTT \\
\hline pMER R & ATGATGCGGCССTCCTGCAGTTAATGGTGATGGTGGTGATGAATGAAATCATAGTCGGATCC \\
\hline BSC483/1a LEU2:: NatMX-f & GATCGTCGTTTTGCCAGGTGACCACGTTGGTCAAGAAATCACACGTACGCTGCAGGTCGAC \\
\hline BSC483/1a LEU2::NatMX-r & СТTAАCTTCTTCGGCGACAGCATCACCGACTTCGGTGGTACTATCGATGAATTCGAGCTCG \\
\hline SUQ5-tet15 & GCTATACTAGTACTCTATCAATGATAGAGTTACCAGATTATTTTGGGCACTATG \\
\hline SUQ5-tetrev & TCATGCTCGAGAAGCTGATATGTTACCGTATG \\
\hline SUQ5-tet15-Sal & GCTATGTCGACACTCTATCAATGATAGAGTTACCAGATTATTTTGGGCACTATG \\
\hline
\end{tabular}


TABLE 2. Saccharomyces cerevisiae strains used in this study

\begin{tabular}{|c|c|}
\hline Strain & Genotype \\
\hline BSC483/1a $\Delta / e u 2$ & $\begin{array}{l}\text { MAT } \alpha \text { SUP45 }{ }^{+} \text {SUQ5 ade2-1 ura3-1 his5-2 lys1-1 can1-100 } \\
\text { PNM1 leu2::NatMX }\end{array}$ \\
\hline sup45-18 $\Delta /$ leu2 & $\begin{array}{l}\text { MAT } \alpha \text { sup45-18 SUQ5 ade2-1 ura3-1 his5-2 lys1-1 can1-100 } \\
\text { PNM1 leu2::NatMX }\end{array}$ \\
\hline sup45-22 $\Delta /$ leu2 & $\begin{array}{l}\text { MAT } \alpha \text { sup45-22 SUQ5 ade2-1 ura3-1 his5-2 lys1-1 can1-100 } \\
\text { PNM1 leu2::NatMX }\end{array}$ \\
\hline sup45-28 $\Delta$ leu2 & $\begin{array}{l}\text { MAT } \alpha \text { sup45-28 SUQ5 ade2-1 ura3-1 his5-2 lys1-1 can1-100 } \\
\text { PNM1 leu2::NatMX }\end{array}$ \\
\hline sup45-42 $\Delta /$ leu2 & $\begin{array}{l}\text { MAT } \alpha \text { sup45-42 SUQ5 ade2-1 ura3-1 his5-2 lys1-1 can1-100 } \\
\text { PNM1 leu2::NatMX }\end{array}$ \\
\hline BY4741 & 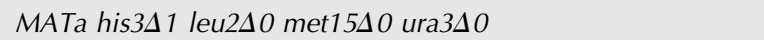 \\
\hline
\end{tabular}

\section{Readthrough assays}

For each readthrough assay construct, three independent transformants were assayed in triplicate. Overnight cultures $(5 \mathrm{~mL})$ were grown to a final $\mathrm{OD}_{600}$ of 0.6. Cells were harvested, washed, resuspended in $150 \mu \mathrm{L}$ of lysis buffer (100 mM potassium phosphate at $\mathrm{pH} 7.8,0.2 \%$ Triton $\mathrm{X}-100)$, lysed using glass beads (400-mesh) in a FastPrep machine (MP Biomedicals) with three cycles of $20 \mathrm{sec}$ of treatment with $5 \mathrm{~min}$ of cooling between each cycle, then centrifuged at $13,000 \mathrm{rpm}$ for $10 \mathrm{~min}$ in a microfuge at $4^{\circ} \mathrm{C}$. The cleared lysate was diluted $1: 5$ in lysis buffer. Of this diluted lysate, $10 \mu \mathrm{L}$ was used in the $\beta$-galactosidase enzyme assay, measured using Galacto-Light Plus System reagents (Applied Biosystems). Luciferase activity was measured using the Bright-Glo Luciferase Assay System (Promega) and $100 \mu \mathrm{L}$ of the diluted lysate. Light emission was measured using a GloMax 96 Microplate Luminometer (Promega). Readthrough was calculated by expressing the mean luciferase/ $\beta$-galactosidase ratio of the test construct as a percentage of the mean luciferase/ $\beta$-galactosidase ratio of the $100 \%$ readthrough control construct, which lacked a stop codon between $l u c$ and lac $Z$ genes.

\section{SDS polyacrylamide gel electrophoresis and Western blot}

Yeast cell lysates were prepared from freshly grown overnight cultures using an alkaline lysis method (von der Haar 2007). SDSPAGE was performed using BioRad Mini-PROTEAN TGK pre-cast gels (12\% polyacrylamide) according to standard protocols (Laemmli 1970). SDS-PAGE gels were semi-dry blotted onto nitrocellulose membranes (GE Healthcare Hybond-C plus; blotted for $15 \mathrm{~min}$ at $15 \mathrm{~V}$, then $15 \mathrm{~min}$ at $10 \mathrm{~V}$ ) and probed using standard protocols (Harlow and Lane 1988). eRF1 was detected using an polyclonal antieRF1 $1^{\circ}$ antibody (1:5000 dilution). Protein loadings were normalized by probing blots with an anti-alcohol dehydrogenase antibody (Abcam \#AB34680; 1:50,000 dilution). Bound conjugated antibodies were detected using SuperSignal West Pico chemiluminescence substrate according to the manufacturer's instructions (Thermo Scientific). Quantification of Western blots was carried out using an Alpha Innotec Fluorchem FC2 charge-coupled device (CCD) camera. Images were captured for 10 frames of $10 \mathrm{sec}$ of exposure, and results were quantified from an appropriately exposed image. Images were manipulated only for the purposes of display; only raw image data were used for quantitative analysis. Background was manually subtracted from all band intensities.

\section{Mathematical model development}

Mathematical models of translation termination developed during the course of this work (Fig. 1), are coarse-grained deterministic models describing a series of "lumped" reactions as detailed (de Silva et al. 2010). Such lumped models can be obtained systematically from more detailed codon-by-codon deterministic models. Model 1 considers translation of the SUP45 mRNA (encoding eRF1) as comprising an initial cap-binding step, followed by "lumped" 5 '-UTR scanning, then a lumped translation elongation step to reach the stop codon. Model 2 describes translation of a generic mRNA containing a premature stop codon. It comprises an initial capbinding step, followed by lumped $5^{\prime}$-UTR scanning, then a lumped translation elongation step to reach the premature stop codon. Following a termination reaction in which competition between the release factor and suppressor tRNA is represented using Michaelis-Menten kinetics (see below), translation elongation to the position of the natural stop codon is again considered as a single lumped rate. Model 3 is identical to Model 2, except that the mRNA being translated is the SUP45 transcript, encoding eRF1. In this model, termination at the initial premature termination codon (PTC) results in the synthesis of an inert, truncated species of eRF1. Termination at the natural stop codon results in the synthesis of a full-length eRF1. Models were implemented using the COPASI modeling software application (Hoops et al. 2006) and are specified in the Supplemental Material. All model files are freely available from the authors.

In Model 2 and Model 3, the competition between suppressor tRNAs and the release factor eRF1 for stop codon recognition was modeled using Michaelis-Menten kinetics (in vitro translation elongation and termination reactions display Michaelis-Mentenlike kinetics) (Pavlov and Ehrenberg 1996), representing the elongation factor ternary complex and the release factor as two substrates competing for a single active site on the ribosome enzyme, using a standard Michaelis-Menten formulation for substrate competition for a single enzyme (Cornish-Bowden 2004). The following two functions (Eqs. 1 and 2) describe that competition, in which each of the substrates eRF1 and SUQ5 acts as an apparent inhibitor term in the function describing the competing reaction.

$$
\begin{gathered}
V_{\text {term }}=\frac{k c a t_{\text {term }} \cdot[T][e R F 1]}{K m_{\text {term }}\left(1+\frac{[S U Q 5]}{K m_{\text {read }}}+\frac{[e R F 1]}{K m_{\text {term }}}\right)} \\
V_{\text {read }}=\frac{k c a t_{\text {read }} \cdot[T] \cdot[S U Q 5]}{K m_{\text {read }}\left(1+\frac{[e R F 1]}{K m_{\text {term }}}+\frac{[S U Q 5]}{K m_{\text {read }}}\right)}
\end{gathered}
$$

Here, $V_{\text {term }}$ and $V_{\text {read }}$ are the velocities of the termination and readthrough reactions, respectively, and $[T],[e R F 1]$, and [SUQ5] are the concentrations, respectively, of ribosomes at the premature stop codon, eRF1, and SUQ5 tRNA, respectively. $K m_{\text {term }}$ and 
TABLE 3. Parameter and constant values used in Models 1,2 , and 3

\begin{tabular}{|c|c|c|}
\hline Parameter & Value & Origin \\
\hline Translation elongation rate & 10 codons/sec & Bonven and Gullov (1979) \\
\hline $5^{\prime}$-Leader $43 \mathrm{~S}$ subunit scanning rate & $10 \mathrm{nt} / \mathrm{sec}$ & Berthelot et al. (2004) \\
\hline $5^{\prime}$-Cap joining rate for $43 \mathrm{~S}$ subunit rate constant & $1.514 \mu \mathrm{M}^{-1} \sec ^{-1}$ & This study; calculated \\
\hline$K_{\text {cat }}$ elongation & $14.9 \mathrm{sec}^{-1}$ & Ruusala et al. (1984) \\
\hline$k_{\mathrm{M}}$ elongation & $0.65 \mu \mathrm{M}$ & Ruusala et al. (1984) \\
\hline$K_{\text {cat }}$ termination & $0.5 \mathrm{sec}^{-1}$ & Freistroffer et al. (2000) \\
\hline$k_{\mathrm{M}}$ termination & $0.008 \mu \mathrm{M}$ & Freistroffer et al. (2000) \\
\hline$K_{\mathrm{M}}$ termination (sup45-18 stop codon context) & $0.0352 \mu \mathrm{M}$ & This study; calculated \\
\hline$K_{\mathrm{M}}$ termination (sup45-22 stop codon context) & $0.0388 \mu \mathrm{M}$ & This study; calculated \\
\hline$K_{\mathrm{M}}$ termination (sup45-28 stop codon context) & $0.0372 \mu \mathrm{M}$ & This study; calculated \\
\hline$K_{\mathrm{M}}$ termination (sup45-42 stop codon context) & $0.0146 \mu \mathrm{M}$ & This study; calculated \\
\hline mRNA depletion through growth rate constant & $0.0001284 \mathrm{sec}^{-1}$ & This study \\
\hline SUP45 mRNA degradation rate constant & $0.001155 \mathrm{sec}^{-1}$ & Wang et al. (2002) \\
\hline SUP45 mRNA synthesis rate & $2.9975 \times 10^{-07} \mu \mathrm{M} / \mathrm{sec}$ & This study; calculated \\
\hline eRF1 protein stability; degradation rate constant & $2.4 \times 10^{-6} \mathrm{sec}^{-1}$ & Moskalenko et al. (2003) \\
\hline SUQ5 mRNA concentration & $0.036 \mu \mathrm{M}$ & This study; calculated \\
\hline Constant & Value & Origin \\
\hline $\begin{array}{l}\text { Concentration of initiation-competent } \\
\text { ribosomes in yeast }\end{array}$ & $0.159 \mu \mathrm{M}$ & $\begin{array}{l}7.6 \% \text { of the } 63,000 \text { elF2 molecules/cell are in } \\
\text { Multi-Function Complexes (Singh et al. 2007) } \\
\text { and assumed here to be loaded on } \\
\text { initiation-competent } 43 \mathrm{~S} \text { subunits. }\end{array}$ \\
\hline eRF1 protein concentration & 13,100 copies per cell & Ghaemmaghami et al. (2003) \\
\hline Length of eRF1 mRNA 5' leader & $21 \mathrm{nt}$ & Nagalakshmi et al. (2008) \\
\hline eRF1 mRNA concentration & $0.00023 \mu \mathrm{M}$ & Beyer et al. (2004) \\
\hline SUP45 ribosomal density & 7 ribosomes/SUP45 mRNA & Arava et al. (2003) \\
\hline Yeast cell volume & $50 \mathrm{fL}$ (average) & Weiss et al. (1975); Johnston et al. (1979) \\
\hline
\end{tabular}

$K m_{\text {read }}$ are the affinity constants for eRF1 and SUQ5 recognition of the stop codon. $k_{c a t} t_{\text {read }}$ and $k_{c a t} t_{\text {term }}$ are the turnover constants, respectively, for the readthrough and termination reactions.

Model 3 also incorporates a description of nonsense-mediated mRNA decay. The position effect of premature nonsense codons in PGK1 mRNA has been experimentally determined with some accuracy (Cao and Parker 2001), showing that $5^{\prime}$-premature stop codons are proportionately more destabilizing than $3^{\prime}$ codons. We made the simplifying assumption that the position effect of nonsense codons on mRNA stability was similar for SUP45 as for PGK1, since this information was not available for SUP45. These data were used to define an NMD destabilization function for incorporation into Model 3. Equation 3 was fitted to the data of Cao and Parker and was used to define the extent of destabilization depending on the relative position of the early stop codon within the mRNA, i.e., the position effect (Eq. 3):

$$
\alpha=0.627 . \beta^{2}-0.0592 \beta+0.328
$$

Here $\alpha$ is the steady-state mRNA stability expressed as a proportion of the wild-type stability, and $\beta$ is the relative position of the premature stop codon, with a $\beta$ value of 1 representing the natural stop codon position.

This experimentally defined NMD function was used to adjust the mRNA degradation rate constants of the sup45-18, -22, -28, and -42 (Table 3 ). However, since stop codon readthrough is known to stabilize the mRNA in several instances-URA3 (Losson and Lacroute 1979), lacZ (Keeling et al. 2004), p53 (Floquet et al. 2011), laminin $\alpha 2$ (Allamand et al. 2008) —in Model 3, the NMD component of the destabilization was dynamically modulated according to the level of readthrough in any system according to Equation 4:

$$
k_{N M D \text { deg }}=k_{N M D}-\left(\frac{[e R F 1]}{[e R F 1]+[e R F 1-t r]} \cdot k_{N M D}\right)+k_{\mathrm{deg}}
$$

Here $k_{\mathrm{NMD}}$ deg is the adjusted, final degradation rate constant for eRF1 mRNA, $k_{\mathrm{NMD}}$ is the NMD-adjusted rate of decay, defined using Equation 3, and $k_{\mathrm{deg}}$ is the rate of eRF1 mRNA turnover in the absence of any NMD effects. For each mutant, the calculated rate constant $k_{\mathrm{NMD}}$ deg was used in the model as the adjusted decay rate for the eRF1 mRNA.

\section{SUPPLEMENTAL MATERIAL}

Supplemental material is available for this article.

\section{ACKNOWLEDGMENTS}

This work was supported by the Biotechnology and Biological Sciences Research Council (BB/G010722/1 to I.S., and BB/I020926/ 1 to I.S. and J.K.).

Received June 21, 2012; accepted September 17, 2012. 


\section{REFERENCES}

Adamski FM, Donly BC, Tate WP. 1993. Competition between frameshifting, termination and suppression at the frameshift site in the Escherichia coli release factor-2 mRNA. Nucleic Acids Res 21: 5074-5078.

Allamand V, Bidou L, Arakawa M, Floquet C, Shiozuka M, PaturneauJouas M, Gartioux C, Butler-Browne GS, Mouly V, Rousset JP, et al. 2008. Drug-induced readthrough of premature stop codons leads to the stabilization of laminin $\alpha 2$ chain mRNA in CMD myotubes. $J$ Gene Med 10: 217-224.

Arava Y, Wang Y, Storey J, Liu C, Brown P, Herschlag D. 2003. Genome-wide analysis of mRNA translation profiles in Saccharomyces cerevisiae. Proc Natl Acad Sci 100: 3889-3894.

Berthelot K, Muldoon M, Rajkowitsch L, Hughes J, McCarthy JE. 2004. Dynamics and processivity of $40 \mathrm{~S}$ ribosome scanning on mRNA in yeast. Mol Microbiol 51: 987-1001.

Bertram G, Innes S, Minella O, Richardson J, Stansfield I. 2001. Endless possibilities: Translation termination and stop codon recognition. Microbiology 147: 255-269.

Betney R, de Silva E, Krishnan J, Stansfield I. 2010. Autoregulatory systems controlling translation factor expression: Thermostat-like control of translational accuracy. RNA 16: 655-663.

Beyer A, Hollunder J, Nasheuer HP, Wilhelm T. 2004. Post-transcriptional expression regulation in the yeast Saccharomyces cerevisiae on a genomic scale. Mol Cell Proteomics 3: 1083-1092.

Bonven B, Gullov K. 1979. Peptide chain elongation rate and ribosomal activity in Saccharomyces cerevisiae as a function of the growth rate. Mol Gen Genet 170: 225-230.

Buchan JR, Aucott LS, Stansfield I. 2006. tRNA properties help shape codon pair preferences in open reading frames. Nucleic Acids Res 34: 1015-1027.

Cao D, Parker R. 2001. Computational modeling of eukaryotic mRNA turnover. RNA 7: 1192-1212.

Chabelskaya S, Kiktev D, Inge-Vechtomov S, Philippe M, Zhouravleva G. 2004. Nonsense mutations in the essential gene SUP35 of Saccharomyces cerevisiae are non-lethal. Mol Genet Genomics 272: 297-307.

Chabelskaya S, Gryzina V, Moskalenko S, Le Goff C, Zhouravleva G. 2007. Inactivation of NMD increases viability of sup 45 nonsense mutants in Saccharomyces cerevisiae. BMC Mol Biol 8: 71. doi: 10.1186/1471-2199-8-71.

Collier J, Binet E, Bouloc P. 2002. Competition between SsrA tagging and translational termination at weak stop codons in Escherichia coli. Mol Microbiol 45: 745-754.

Cornish-Bowden A. 2004. Fundamentals of enzyme kinetics. Portland Press, London.

Craigen WJ, Caskey CT. 1986. Expression of peptide chain release factor 2 requires high-efficiency frameshift. Nature 322: 273-275.

Curran JF, Yarus M. 1988. Use of tRNA suppressors to probe regulation of Escherichia coli release factor 2. J Mol Biol 203: 75-83.

de Silva E, Krishnan J, Betney R, Stansfield I. 2010. A mathematical modelling framework for elucidating the role of feedback control in translation termination. J Theor Biol 264: 808-821.

Donly BC, Edgar CD, Adamski FM, Tate WP. 1990. Frameshift autoregulation in the gene for Escherichia coli release factor 2: Partly functional mutants result in frameshift enhancement. Nucleic Acids Res 18: 6517-6522.

Edelman I, Culbertson MR. 1991. Exceptional codon recognition by the glutamine tRNAs in Saccharomyces cerevisiae. EMBO J 10: 1481-1491.

Eliseev B, Kryuchkova P, Alkalaeva E, Frolova L. 2011. A single amino acid change of translation termination factor eRF1 switches between bipotent and omnipotent stop-codon specificity. Nucleic Acids Res 39: 599-608.

Floquet C, Deforges J, Rousset JP, Bidou L. 2011. Rescue of non-sense mutated p53 tumor suppressor gene by aminoglycosides. Nucleic Acids Res 39: 3350-3362.

Freistroffer DV, Pavlov MY, MacDougall J, Buckingham RH, Ehrenberg M. 1997. Release factor RF3 in E. coli accelerates the dissociation of release factors RF1 and RF2 from the ribosome in a GTP-dependent manner. EMBO J 16: 4126-4133.

Freistroffer DV, Kwiatkowski M, Buckingham RH, Ehrenberg M. 2000. The accuracy of codon recognition by polypeptide release factors. Proc Natl Acad Sci 97: 2046-2051.

Frolova L, Le Goff X, Rasmussen HH, Cheperegin S, Drugeon G, Kress M, Arman I, Haenni AL, Celis JE, Philippe M. 1994. A highly conserved eukaryotic protein family possessing properties of polypeptide chain release factor. Nature 372: 701-703.

Gao H, Zhou Z, Rawat U, Huang C, Bouakaz L, Wang C, Cheng Z, Liu Y, Zavialov A, Gursky R, et al. 2007. RF3 induces ribosomal conformational changes responsible for dissociation of class I release factors. Cell 129: 929-941.

Gari E, Piedrafita L, Aldea M, Herrero E. 1997. A set of vectors with a tetracycline-regulatable promoter system for modulated gene expression in Saccharomyces cerevisiae. Yeast 13: 837-848.

Ghaemmaghami S, Huh WK, Bower K, Howson RW, Belle A, Dephoure N, O'Shea EK, Weissman JS. 2003. Global analysis of protein expression in yeast. Nature 425: 737-741.

Golding I, Paulsson J, Zawilski SM, Cox EC. 2005. Real-time kinetics of gene activity in individual bacteria. Cell 123: 1025-1036.

Harlow E, Lane D. 1988. Antibodies: A laboratory manual. Cold Spring Harbor Laboratory, Cold Spring Harbor, NY.

Hayes CS, Bose B, Sauer RT. 2002a. Proline residues at the C terminus of nascent chains induce SsrA tagging during translation termination. J Biol Chem 277: 33825-33832.

Hayes CS, Bose B, Sauer RT. 2002b. Stop codons preceded by rare arginine codons are efficient determinants of SsrA tagging in Escherichia coli. Proc Natl Acad Sci 99: 3440-3445.

Hoops S, Sahle S, Gauges R, Lee C, Pahle J, Simus N, Singhal M, Xu L, Mendes P, Kummer U. 2006. COPASI-a COmplex PAthway SImulator. Bioinformatics 22: 3067-3074.

Johnston GC, Ehrhardt CW, Lorincz A, Carter BL. 1979. Regulation of cell size in the yeast Saccharomyces cerevisiae. J Bacteriol 137: 1-5.

Keeling KM, Lanier J, Du M, Salas-Marco J, Gao L, Kaenjak-Angeletti A, Bedwell DM. 2004. Leaky termination at premature stop codons antagonizes nonsense-mediated mRNA decay in S. cerevisiae. RNA 10: 691-703.

Kiktev D, Moskalenko S, Murina O, Baudin-Baillieu A, Rousset JP, Zhouravleva G. 2009. The paradox of viable sup45 STOP mutations: A necessary equilibrium between translational readthrough, activity and stability of the protein. Mol Genet Genomics 282: 83-96.

Laemmli UK. 1970. Cleavage of structural proteins during the assembly of the head of bacteriophage T4. Nature 227: 680-685.

Losson R, Lacroute F. 1979. Interference of nonsense mutations with eukaryotic messenger RNA stability. Proc Natl Acad Sci 76: 51345137.

Major LL, Poole ES, Dalphin ME, Mannering SA, Tate WP. 1996. Is the in-frame termination signal of the Escherichia coli release factor-2 frameshift site weakened by a particularly poor context? Nucleic Acids Res 24: 2673-2678.

Martin R, Weiner M, Gallant J. 1988. Effects of release factor context at UAA codons in Escherichia coli. J Bacteriol 170: 4714-4717.

Mikuni O, Kawakami K, Nakamura Y. 1991. Sequence and functional analysis of mutations in the gene encoding peptide-chain-release factor 2 of Escherichia coli. Biochimie 73: 1509-1516.

Moskalenko SE, Chabelskaya SV, Inge-Vechtomov SG, Philippe M, Zhouravleva GA. 2003. Viable nonsense mutants for the essential gene SUP45 of Saccharomyces cerevisiae. BMC Mol Biol 4: 2. doi: 10.1186/1471-2199-4-2.

Nagalakshmi U, Wang Z, Waern K, Shou C, Raha D, Gerstein M, Snyder M. 2008. The transcriptional landscape of the yeast genome defined by RNA sequencing. Science 320: 1344-1349.

Pavlov MY, Ehrenberg M. 1996. Rate of translation of natural mRNAs in an optimized in vitro system. Arch Biochem Biophys 328: 9-16.

Pavlov MY, Freistroffer DV, Dincbas V, MacDougall J, Buckingham RH, Ehrenberg M. 1998. A direct estimation of the context effect on the efficiency of termination. J Mol Biol 284: 579-590. 
Percudani R, Pavesi A, Ottonello S. 1997. Transfer RNA gene redundancy and translational selection in Saccharomyces cerevisiae. J Mol Biol 268: 322-330.

Poole ES, Brown CM, Tate WP. 1995. The identity of the base following the stop codon determines the efficiency of in vivo translational termination in Escherichia coli. EMBO J 14: 151158.

Pure GA, Robinson GW, Naumovski L, Friedberg EC. 1985. Partial suppression of an ochre mutation in Saccharomyces cerevisiae by multicopy plasmids containing a normal yeast tRNA ${ }^{\text {Gln }}$ gene. J Mol Biol 183: 31-42.

Ruusala T, Andersson D, Ehrenberg M, Kurland CG. 1984. Hyperaccurate ribosomes inhibit growth. EMBO J 3: 2575-2580.

Sambrook J, Russell DW. 2001. Molecular cloning: A laboratory manual, 3rd ed. Cold Spring Harbor Laboratory Press, Cold Spring Harbor, NY.

Sikorski RS, Hieter P. 1989. A system of shuttle vectors and yeast host strains designed for efficient manipulation of DNA in Saccharomyces cerevisiae. Genetics 122: 19-27.

Singh CR, Udagawa T, Lee B, Wassink S, He H, Yamamoto Y, Anderson JT, Pavitt GD, Asano K. 2007. Change in nutritional status modulates the abundance of critical pre-initiation intermediate complexes during translation initiation in vivo. J Mol Biol 370: $315-330$.

Stahl G, Bidou L, Rousset JP, Cassan M. 1995. Versatile vectors to study recoding: Conservation of rules between yeast and mammalian cells. Nucleic Acids Res 23: 1557-1560.

Stansfield I, Jones KM, Kushnirov VV, Dagkesamanskaya AR, Poznyakovski AI, Paushkin SV, Nierras CR, Cox BS, TerAvanesyan MD, Tuite MF. 1995a. The products of the SUP45 (eRF1) and SUP35 genes interact to mediate translation termination in Saccharomyces cerevisiae. EMBO J 14: 4365-4373.

Stansfield I, Akhmaloka, Tuite MF. 1995b. A mutant allele of the SUP45 (SAL4) gene of Saccharomyces cerevisiae shows temperature-de- pendent allosuppressor and omnipotent suppressor phenotypes. Curr Genet 27: 417-426.

Stansfield I, Eurwilaichitr L, Akhmaloka, Tuite MF. 1996. Depletion in the levels of the release factor eRF1 causes a reduction in the efficiency of translation termination in yeast. Mol Microbiol 20: $1135-1143$.

Sunohara T, Jojima K, Yamamoto Y, Inada T, Aiba H. 2004. Nascentpeptide-mediated ribosome stalling at a stop codon induces mRNA cleavage resulting in nonstop mRNA that is recognized by tmRNA. RNA 10: 378-386.

von der Haar T. 2007. Optimized protein extraction for quantitative proteomics of yeasts. PLoS ONE 2: e1078. doi: 10.1371/journal. pone. 0001078 .

Waldron C, Lacroute F. 1975. Effect of growth rate on the amounts of ribosomal and transfer ribonucleic acids in yeast. $J$ Bacteriol 122: 855-865.

Waldron C, Cox BS, Wills N, Gesteland RF, Piper PW, Colby D, Guthrie C. 1981. Yeast ochre suppressor SUQ5-ol is an altered

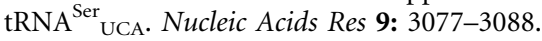

Wang Y, Liu C, Storey J, Tibshirani R, Herschlag D, Brown P. 2002. Precision and functional specificity in mRNA decay. Proc Natl Acad Sci 99: 5860-5865.

Weiss RL, Kukora JR, Adams J. 1975. The relationship between enzyme activity, cell geometry, and fitness in Saccharomyces cerevisiae. Proc Natl Acad Sci 72: 794-798.

Williams I, Richardson J, Starkey A, Stansfield I. 2004. Genome-wide prediction of stop codon readthrough during translation in the yeast Saccharomyces cerevisiae. Nucleic Acids Res 32: 6605-6616.

Zaher HS, Green R. 2009. Quality control by the ribosome following peptide bond formation. Nature 457: 161-166.

Zhouravleva G, Frolova L, Le Goff X, Le Guellec R, Inge-Vechtomov S, Kisselev L, Philippe M. 1995. Termination of translation in eukaryotes is governed by two interacting polypeptide chain release factors, eRF1 and eRF3. EMBO J 14: 4065-4072. 

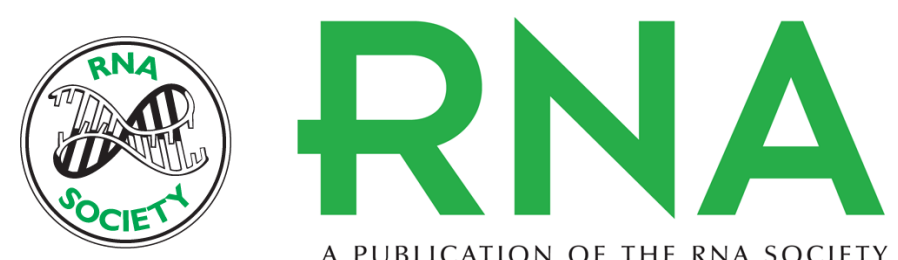

A PUBLICATION OF THE RNA SOCIETY

\section{Regulation of release factor expression using a translational negative feedback loop: A systems analysis}

Russell Betney, Eric de Silva, Christina Mertens, et al.

RNA 2012 18: 2320-2334 originally published online October 25, 2012

Access the most recent version at doi:10.1261/rna.035113.112

\section{Supplemental http://rnajournal.cshlp.org/content/suppl/2012/10/15/rna.035113.112.DC1 \\ Material}

References This article cites 61 articles, 17 of which can be accessed free at:

http://rnajournal.cshlp.org/content/18/12/2320.full.html\#ref-list-1

\section{License}

Email Alerting Receive free email alerts when new articles cite this article - sign up in the box at the Service top right corner of the article or click here.

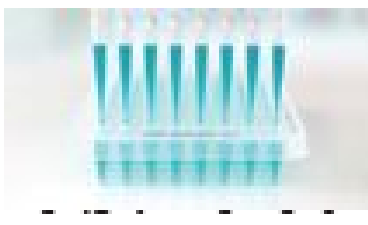

Providing Precise Solutions for your research.

To subscribe to RNA go to:

http://rnajournal.cshlp.org/subscriptions 